\title{
Evolutionary synthesis of simple stellar populations
}

\section{Colours and indices}

\author{
O.M. Kurth, U. Fritze - v. Alvensleben, and K.J. Fricke \\ Universitätssternwarte Göttingen, Geismarlandstr. 11, 37083 Göttingen, Germany \\ e-mail: okurth@uni-sw.gwdg.de
}

Received March 16, 1998; accepted February 11, 1999

\begin{abstract}
We construct evolutionary synthesis models for simple stellar populations using the evolutionary tracks from the Padova group $(1993,1994)$, theoretical colour calibrations from Lejeune et al. $(1997,1998)$ and fit functions for stellar atmospheric indices from Worthey et al. (1994).

A Monte-Carlo technique allows us to obtain a smooth time evolution of both broad band colours in UBVRIK and a series of stellar absorption features for Single Burst Stellar Populations (SSPs). We present colours and indices for SSPs with ages from $110^{9}$ yrs to $1.610^{10}$ yrs and metallicities $[M / H]=-2.3,-1.7,-0.7,-0.4,0.0$ and 0.4 .

Model colours and indices at an age of about a Hubble time are in good agreement with observed colours and indices of the Galactic and M 31 GCs.
\end{abstract}

Key words: Galaxy: globular cluster; open clusters galaxies: starclusters; M 31; evolution

\section{Introduction}

Colour distributions of Globular Cluster (GC) systems are observed for a large number of early-type galaxies (E, S0, dE, cD) using ground-based Washington or HST broad band photometry. In most cases double-peak or broad/multi-peak colour distributions are seen (e.g. Zepf \& Ashman 1992; Elson \& Santiago 1996; Kissler-Patig et al. 1997).

If the different colour subpopulations of GCs are formed in different events then they may contain clues to the formation history of their parent galaxies. For example a two-peak colour distribution may result, if in addition to a primary initial collapse population of

Send offprint requests to: O. Kurth

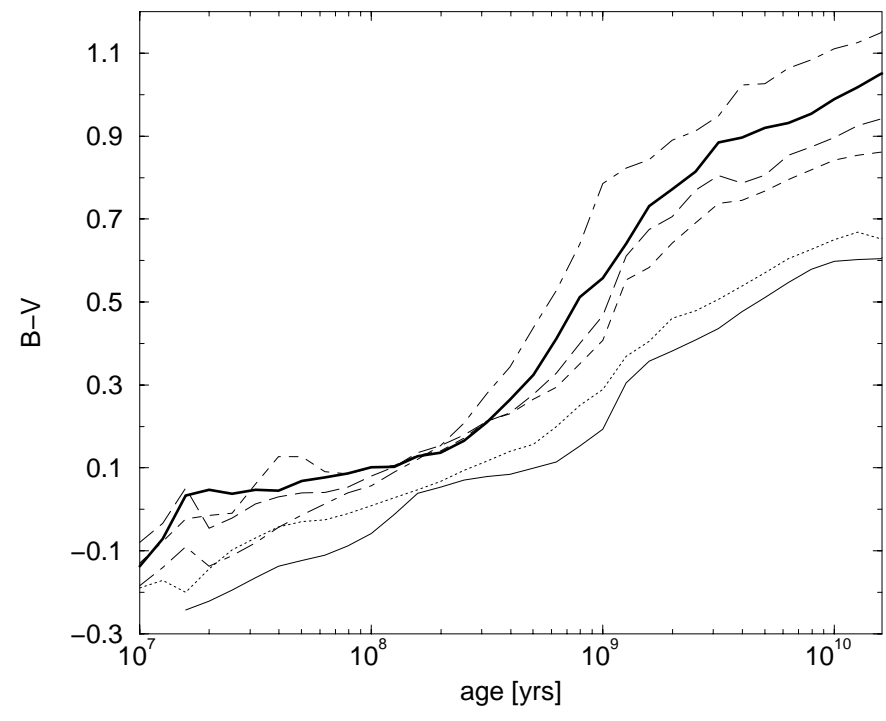

Fig. 1. $B-V$ versus time with logarithmic scaling for time (solid line: $Z=0.0001$, dotted line: $Z=0.0004$, short-dashed line: $Z=0.004$, long dashed line: $Z=0.008$, thick line: $Z=0.02$, dot-dashed line: $Z=0.05$ )

GCs, a secondary population of GCs were formed either in a merger-induced starburst (Schweizer 1993; Ashman \& Zepf 1992; Fritze - v. Alvensleben \& Gerhard 1994; Fritze - v. Alvensleben \& Burkert 1995) or else in some distinct secondary phase of cluster formation within the original galaxy (Forbes et al. 1997). Likewise the broad or multi-peaked colour distribution often observed in GC systems around $\mathrm{cD}$ galaxies may point to a series of $\mathrm{GC}$ formation events during the hierarchical assembly of the parent galaxy or to some protracted GC formation or accretion mechanism.

A well-known difficulty with the interpretation of colour distributions is the degeneracy of colours with respect to age and metallicity. While for Washington photometry there are well established and reliable calibrations of colours in terms of metallicity, the situation 


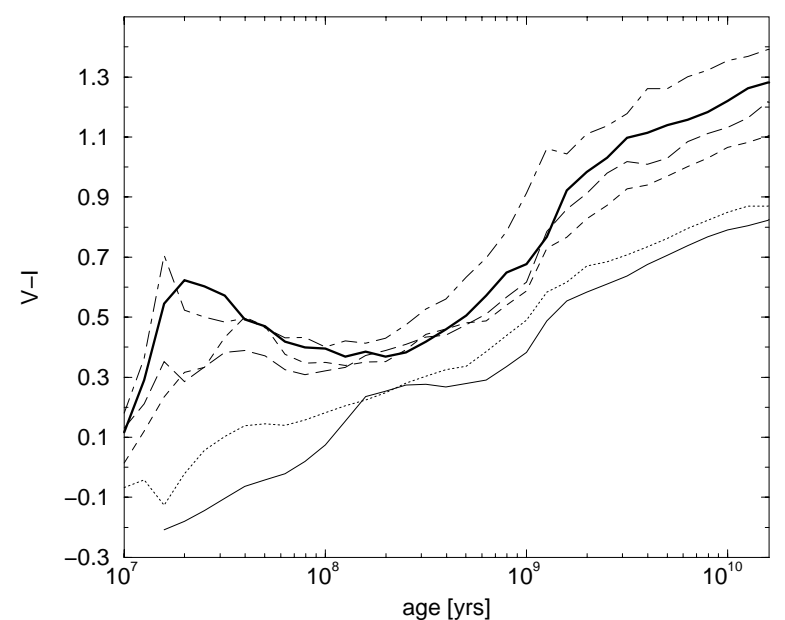

Fig. 2. $V-I$ versus time with logarithmic scaling for time

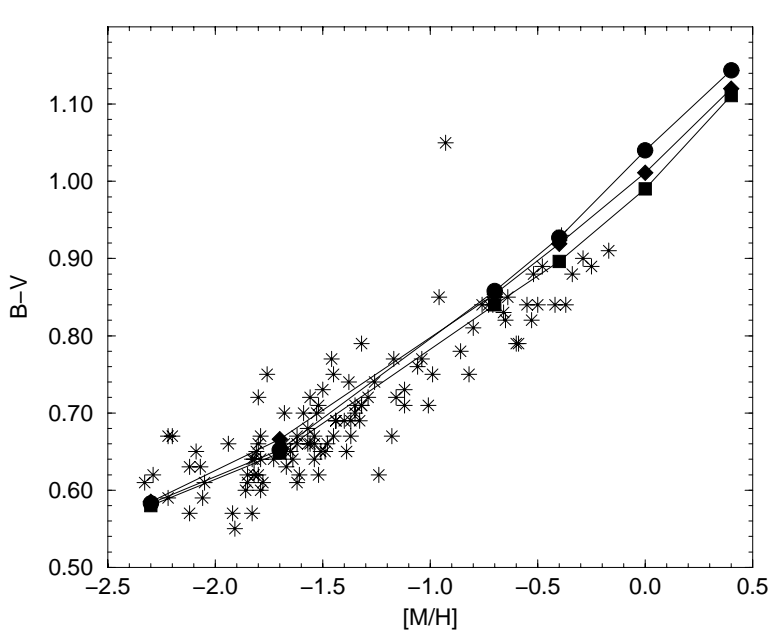

Fig. 3. $B-V$ colour versus metallicity for observerd clusters (stars) from Harris with $E(B-V)<0.4$ and models at 10 (squares),12 (diamonds) and 15 (circles) Gyrs with $\eta=0.35$. The observed colours have been deredened

with HST broad band observations of GC systems is less clear. A better understanding of the formation of composite GC systems would be possible if separate age and metallicity distributions could be disentangled from an observed colour distribution.

A second issue concerns the interpretation of colours for young star cluster systems detected with HST in many interacting and starburst galaxies. The question is, if these YSC systems - at least some fraction of them - are the progenitors of GC systems. In an attempt to answer this question star clusters are being imaged with HST in an age sequence of interacting galaxies - from early stages of interaction through merger remnants up to E/S0s

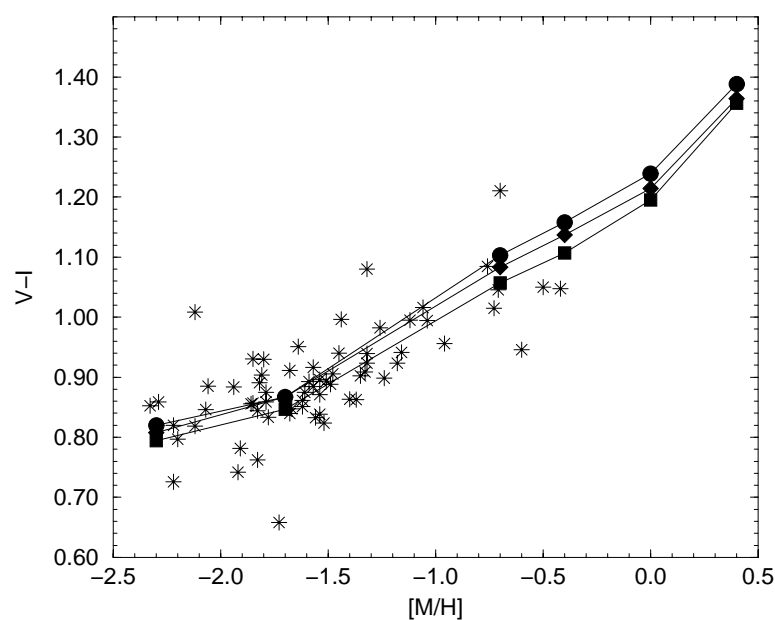

Fig. 4. Same as Fig. 3, but for $V-I$

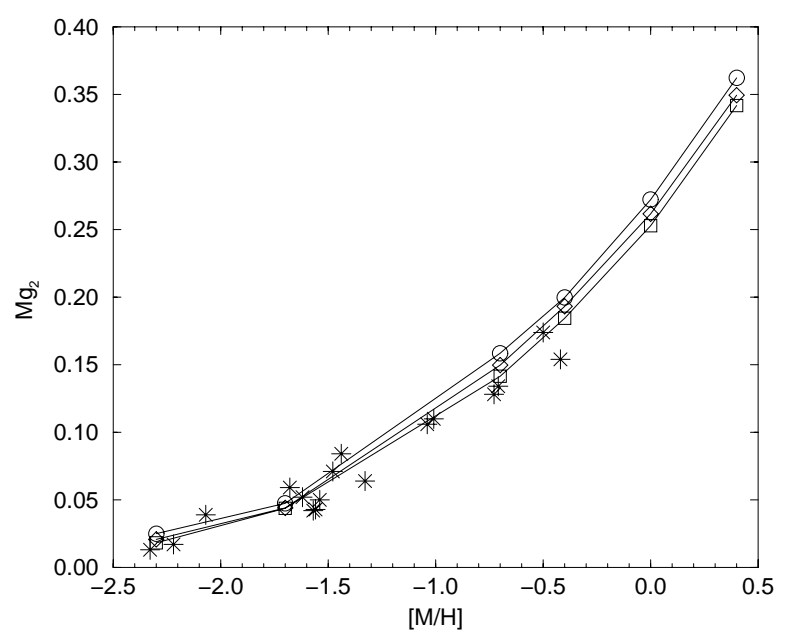

Fig. 5. $\mathrm{Mg}_{2}$ versus metallicity for observerd clusters (stars) and models at 10 (squares), 12 (diamonds) and 15 (circles) Gyrs with $\eta=0.35$. The observed indices are from Burstein, their metallicities from Harris

(e.g. Schweizer et al. 1996; Whitmore et al. 1995; Miller et al. 1997). With $10 \mathrm{~m}$ class telescopes, spectroscopy of the brighter members of young star cluster populations is becoming possible (Kissler-Patig et al. 1998; Brodie et al. 1998 , but see also $4-5 \mathrm{~m}$ class spectra by Schweizer \& Seitzer 1993 or Zepf et al. 1995). Spectroscopy will only be possible for a subsample of YSCs. Thus the determination of ages and metallicities from broad band colors will still be necessary.

It is thus desirable to study the evolution of broad band colours and absorption indices for single burst stellar populations of various metallicities using the most recent and complete stellar evolutionary tracks as well as 


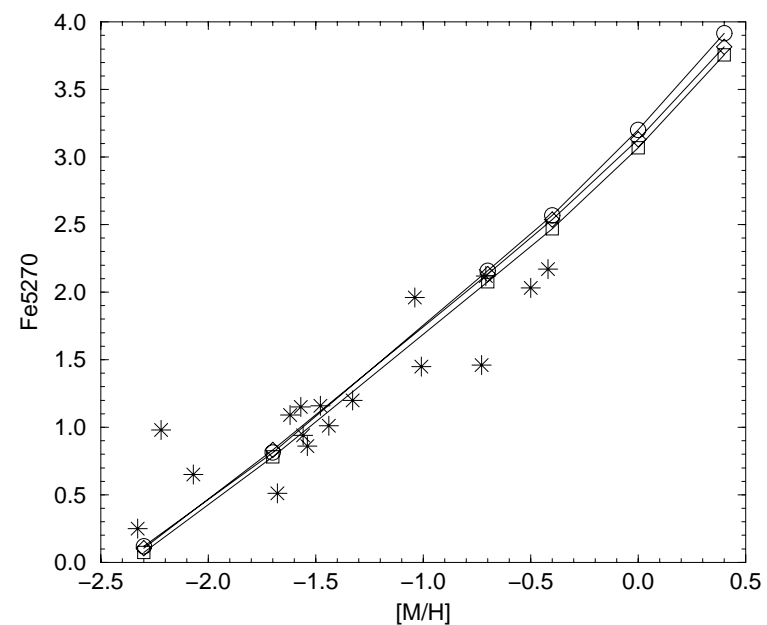

Fig. 6. Same as Fig. 5, but for Fe5270

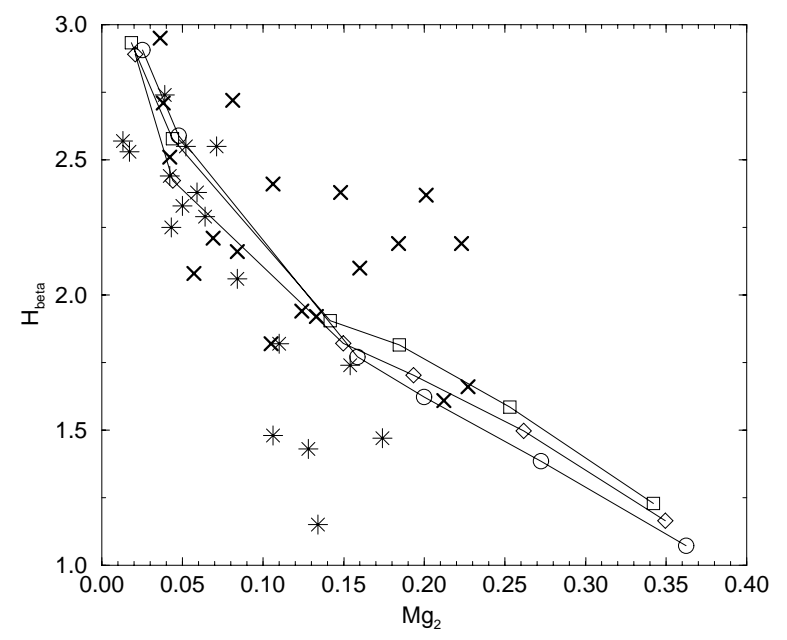

Fig. 7. The $\mathrm{H}_{\beta}$ index against $\mathrm{Mg}_{2}$ index for observations of Galactic GCs (stars), M 31 GCs (crosses) and models

careful colour and index calibrations. This allows one to obtain theoretical calibrations of broad band colours and indices in terms of metallicity over the full range of ages under investigation, i.e. from $10^{7}$ yr to a Hubble time.

Since theoretical calculations for the evolution of stars are only available for a discrete grid of masses, some means for obtaining a smooth evolution of the composite population is needed. Applying the tracks as they are would create discontinuities because all stars of a given mass would reach the giant branch at the same time, dominating the integrated light until they die. This effect is large for populations with stars that have about the same age. The effect also increases with age of the whole population, since the differences in both the lifetimes and luminosities

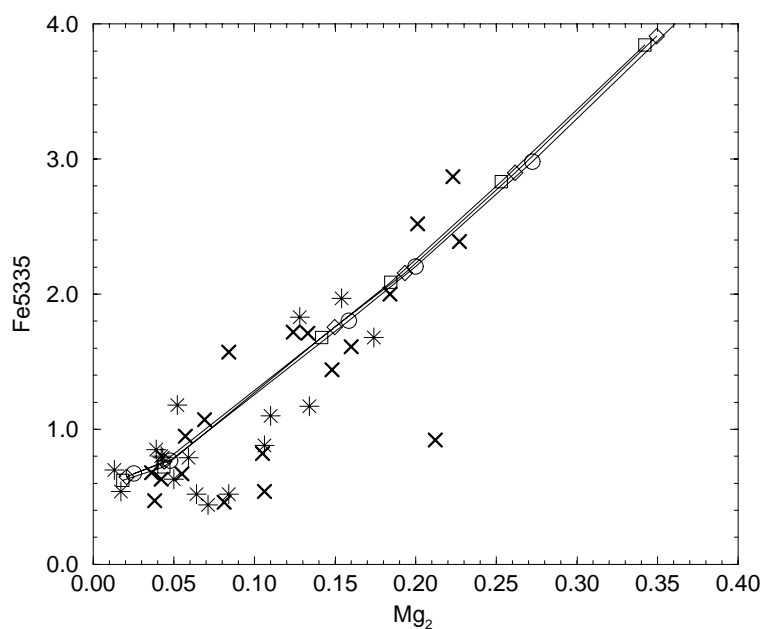

Fig. 8. Same as Fig. 7 but for the Fe5335 index

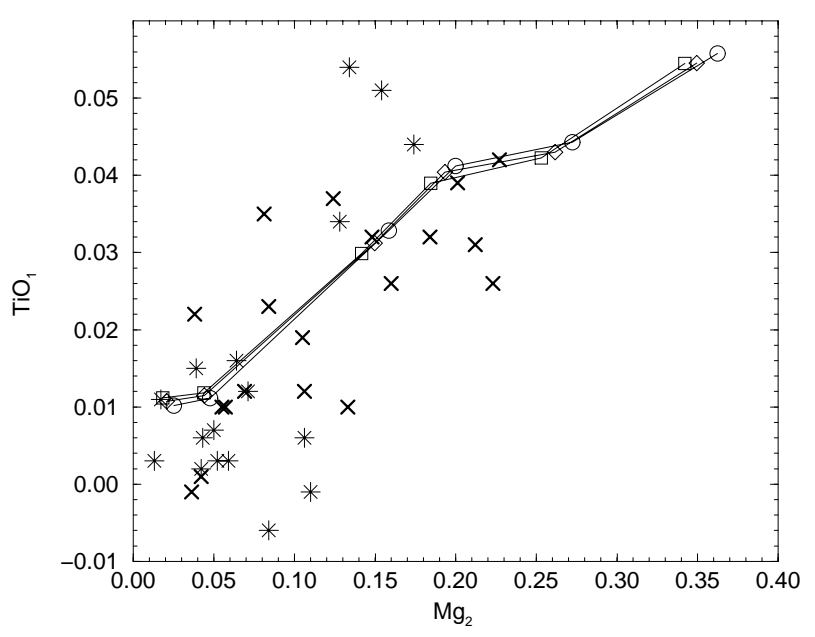

Fig. 9. Same as Fig. 7 but for the $\mathrm{TiO}_{1}$ index

between the main sequence and the later stages increase with decreasing mass.

For this work, we use the Monte Carlo method to bypass this problem while still avoiding the interpolation of tracks with its accompanying danger of creating artificial states. This is described in detail in Sect. 2.3.

The star formation history of any stellar system can be described by a superposition of SSP models of different ages and metallicities. An example of this is given by Cellone \& Forte et al. (1996) in their study of Low Surface Brightness galaxies or Contardo et al. (1998) who investigate the formation and evolution of galaxies in a cosmological scenario. 


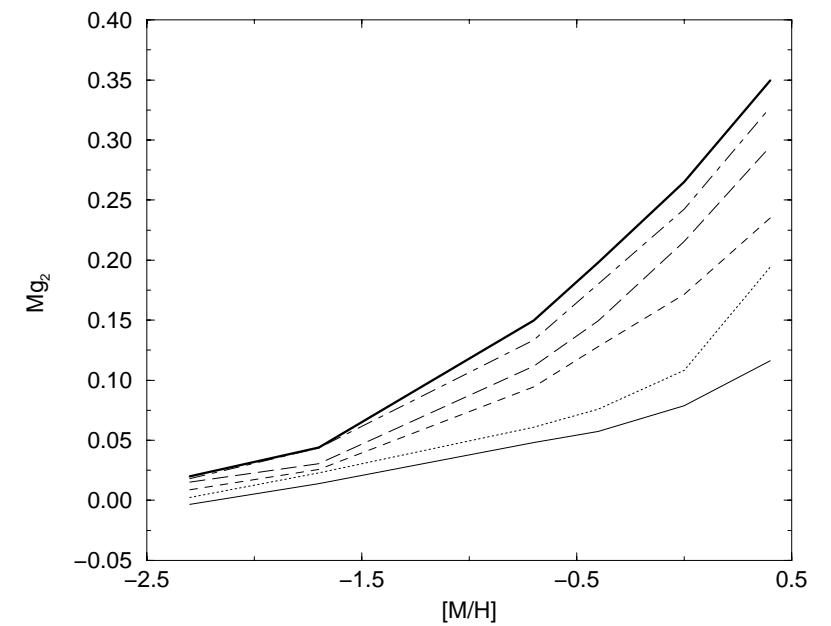

Fig. 10. The $\mathrm{Mg}_{2}$ index against metallicity for various ages. Thin solid line: 0.5 Gyrs, dotted line: 1 Gyr, dashed: 2 Gyrs, long dashed: 4 Gyrs, dot dashed: 8 Gyrs, thick solid line: 12 Gyrs

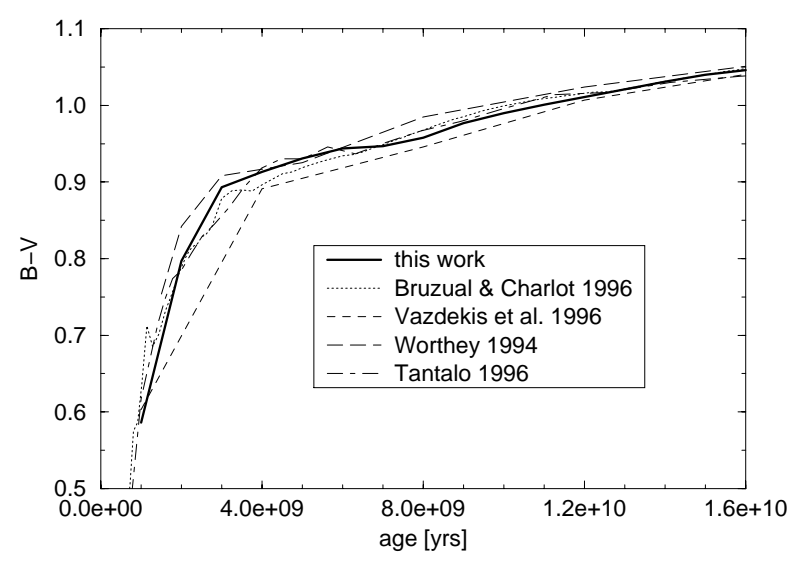

Fig. 11. Comparison of $B-V$ against age predicted with our models with those of other authors

\section{Model description}

\subsection{Input physics}

We use the evolutionary tracks of the Padova group (Bressan et al. 1993; Fagotto et al. 1994a, 1994b, 1994c; Hereafter referred to as the Padova tracks). The Padova group gives effective temperatures and luminosities as a function of time for many masses $\left(0.6-120 M_{\odot}\right)$ and a metallicity range from $Z=0.0001$ to $Z=0.05$. Their tracks include all stages in stellar evolution from the zero age main sequence to the tip of the RGB and from the zero age horizontal branch to the tip of the EAGB. For the stellar mass loss we use the method described in Bressan et al. (1993) with Reimers law.

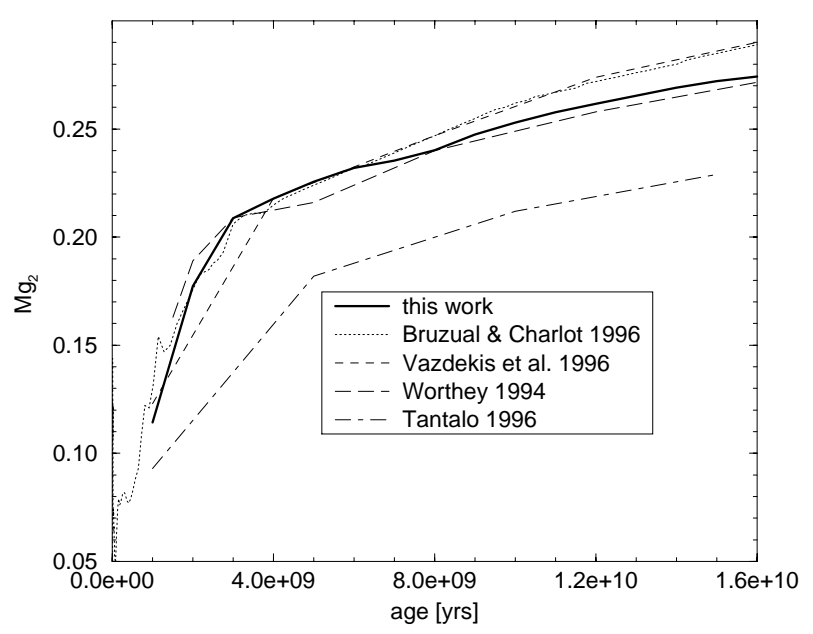

Fig. 12. Comparison of the $\mathrm{Mg}_{2}$ index against age as predicted with our models to those of other authors

For lower masses $\left(0.08 M_{\odot}\right.$ to $\left.0.5 M_{\odot}\right)$ we use the calculations from Chabrier \& Baraffe (1997). They based their calculation on a new description of the interiour of low mass objects and use non-grey atmospheres. Since their grid in metallicity does not match the grid of the Padova group we have linearly interpolated the values for $[M / H]=-1.7,[M / H]=-0.7$ and $[M / H]=-0.4$ while for $[M / H]=-2.3$ and $[M / H]=0.4$ we used the calculations for $[M / H]=-2.0$ and $[M / H]=0$, respectively. The contributions of these low mass stars to the integrated light is very low, therefore the error should be small.

To obtain synthetic colours, the conversion from theoretical quantities to observable quantities is very important. The evolutionary tracks for stars give effective temperatures, bolometric luminosities and gravities at the surface of stars of different masses as a function of time. These values have to be converted to colours in the various bands and to atmospheric indices of the evolutionary states.

For the fluxes and colours we use the theoretical library of model atmosphere spectra for various metallicities of Lejeune et al. (1997, 1998) Lejeune et al. have assembled a coherent library of synthetic stellar atmosphere calculations from Kurucz (see e.g. Kurucz 1979); Fluks et al. (1994); Bessell et al. (1989, 1991). The library has an effective temperature range from $T_{\text {eff }}=2000 \mathrm{~K}$ to $50000 \mathrm{~K}$ and covers a broad range of metallicities. To cope with discrepancies between colours derived from model atmosphere spectra and observed colours, they correct (i.e. bend) the model spectra to give agreement with observed colours for $U$ through $K$. To fit their metallicity grid to that of the stellar evolutionary tracks, colours and bolometric corrections are interpolated linearly.

For a series of absorption indices, the empirical functions of Worthey et al. (1994) are used. Worthey et al. 
Table 1. The model colours. Time is in years. $U, B$ and $V$ are in the Johnson system, $R$ and $I$ in the Cousins system, $K$ as in Bessell \& Brett (1988)

\begin{tabular}{|c|c|c|c|c|c|}
\hline time & $\overline{U-B}$ & $B-V$ & $V-R$ & $V-I$ & $V-K$ \\
\hline \multicolumn{6}{|c|}{$Z=0.0001$} \\
\hline $1.010^{7}$ & -0.80 & 0.05 & 0.14 & 0.34 & 0.99 \\
\hline $2.010^{7}$ & -0.90 & -0.22 & -0.09 & -0.18 & -0.41 \\
\hline $3.010^{7}$ & -0.76 & -0.17 & -0.06 & -0.11 & -0.19 \\
\hline $4.010^{7}$ & -0.65 & -0.14 & -0.04 & -0.06 & -0.03 \\
\hline $5.010^{7}$ & -0.60 & -0.12 & -0.03 & -0.04 & 0.03 \\
\hline $6.010^{7}$ & -0.57 & -0.11 & -0.03 & -0.03 & 0.06 \\
\hline $7.010^{7}$ & -0.55 & -0.10 & -0.02 & -0.01 & 0.15 \\
\hline $8.010^{7}$ & -0.53 & -0.09 & -0.01 & 0.02 & 0.23 \\
\hline $9.010^{7}$ & -0.51 & -0.07 & 0.01 & 0.05 & 0.32 \\
\hline $1.010^{8}$ & -0.49 & -0.06 & 0.02 & 0.07 & 0.40 \\
\hline $2.010^{8}$ & -0.39 & 0.06 & 0.10 & 0.25 & 0.88 \\
\hline $3.010^{8}$ & -0.30 & 0.08 & 0.11 & 0.27 & 0.91 \\
\hline $4.010^{8}$ & -0.21 & 0.08 & 0.11 & 0.27 & 0.89 \\
\hline $5.010^{8}$ & -0.17 & 0.10 & 0.11 & 0.28 & 0.91 \\
\hline $6.010^{8}$ & -0.12 & 0.11 & 0.12 & 0.29 & 0.91 \\
\hline $7.010^{8}$ & -0.09 & 0.11 & 0.12 & 0.28 & 0.88 \\
\hline $8.010^{8}$ & -0.06 & 0.15 & 0.14 & 0.34 & 0.99 \\
\hline $9.010^{8}$ & -0.02 & 0.10 & 0.09 & 0.23 & 0.74 \\
\hline $1.010^{9}$ & -0.08 & 0.19 & 0.16 & 0.38 & 1.11 \\
\hline $1.010^{9}$ & -0.08 & 0.19 & 0.16 & 0.38 & 1.11 \\
\hline $2.010^{9}$ & -0.02 & 0.38 & 0.27 & 0.58 & 1.51 \\
\hline $3.010^{9}$ & -0.01 & 0.43 & 0.29 & 0.63 & 1.59 \\
\hline $4.010^{9}$ & -0.01 & 0.48 & 0.32 & 0.68 & 1.67 \\
\hline $5.010^{9}$ & -0.01 & 0.51 & 0.34 & 0.71 & 1.73 \\
\hline $6.010^{9}$ & -0.02 & 0.54 & 0.35 & 0.73 & 1.77 \\
\hline $7.010^{9}$ & -0.02 & 0.56 & 0.36 & 0.75 & 1.81 \\
\hline $8.010^{9}$ & -0.03 & 0.58 & 0.37 & 0.77 & 1.84 \\
\hline $9.010^{9}$ & -0.03 & 0.59 & 0.38 & 0.78 & 1.86 \\
\hline $1.010^{10}$ & -0.02 & 0.60 & 0.38 & 0.79 & 1.88 \\
\hline $1.110^{10}$ & -0.02 & 0.60 & 0.38 & 0.80 & 1.90 \\
\hline $1.210^{10}$ & -0.01 & 0.60 & 0.39 & 0.80 & 1.91 \\
\hline $1.310^{10}$ & 0.00 & 0.60 & 0.39 & 0.81 & 1.93 \\
\hline $1.410^{10}$ & 0.00 & 0.60 & 0.39 & 0.81 & 1.93 \\
\hline $1.510^{10}$ & 0.00 & 0.60 & 0.39 & 0.81 & 1.95 \\
\hline $1.610^{10}$ & -0.01 & 0.59 & 0.39 & 0.82 & 1.95 \\
\hline
\end{tabular}

supply fitting functions that give index strength as a function of $T_{\text {eff }}, \log g$, and $[\mathrm{Fe} / \mathrm{H}]$. These were obtained from observations of 460 stars, covering a large range in the above parameters.

\subsection{Model parameters}

Once the input physics database is defined, the only free parameters in an evolutionary synthesis calculation for an SSP are those describing the initial mass function IMF.

The IMF can be expressed as:

$\phi(m) \mathrm{d} m \propto m^{-(1+x)} \mathrm{d} m$

where the precise exponent $x$ is observationally still somewhat controversal. For globular clusters, Chabrier \& Méra
Table 2. Continuation of Table 1 for $Z=0.0004$

\begin{tabular}{|c|c|c|c|c|c|}
\hline time & $\overline{U-B}$ & $B-V$ & $V-R$ & $\overline{V-I}$ & $V-K$ \\
\hline \multicolumn{6}{|c|}{$Z=0.0004$} \\
\hline $1.010^{7}$ & -0.96 & -0.19 & -0.04 & -0.07 & -0.01 \\
\hline $2.010^{7}$ & -0.80 & -0.14 & -0.03 & -0.02 & 0.21 \\
\hline $3.010^{7}$ & -0.67 & -0.08 & 0.02 & 0.09 & 0.55 \\
\hline $4.010^{7}$ & -0.59 & -0.04 & 0.05 & 0.14 & 0.66 \\
\hline $5.010^{7}$ & -0.54 & -0.03 & 0.05 & 0.14 & 0.66 \\
\hline $6.010^{7}$ & -0.51 & -0.03 & 0.05 & 0.14 & 0.62 \\
\hline $7.010^{7}$ & -0.47 & -0.02 & 0.05 & 0.15 & 0.64 \\
\hline $8.010^{7}$ & -0.44 & -0.01 & 0.06 & 0.16 & 0.66 \\
\hline $9.010^{7}$ & -0.41 & 0.00 & 0.06 & 0.17 & 0.69 \\
\hline $1.010^{8}$ & -0.39 & 0.01 & 0.07 & 0.18 & 0.71 \\
\hline $2.010^{8}$ & -0.25 & 0.07 & 0.10 & 0.25 & 0.85 \\
\hline $3.010^{8}$ & -0.16 & 0.11 & 0.12 & 0.30 & 0.95 \\
\hline $4.010^{8}$ & -0.11 & 0.14 & 0.14 & 0.33 & 0.99 \\
\hline $5.010^{8}$ & -0.10 & 0.16 & 0.14 & 0.34 & 1.00 \\
\hline $6.010^{8}$ & -0.09 & 0.19 & 0.16 & 0.37 & 1.06 \\
\hline $7.010^{8}$ & -0.09 & 0.22 & 0.18 & 0.41 & 1.12 \\
\hline $8.010^{8}$ & -0.09 & 0.25 & 0.20 & 0.44 & 1.17 \\
\hline $9.010^{8}$ & -0.09 & 0.27 & 0.21 & 0.47 & 1.23 \\
\hline $1.010^{9}$ & -0.10 & 0.29 & 0.22 & 0.49 & 1.26 \\
\hline $1.010^{9}$ & -0.10 & 0.29 & 0.22 & 0.49 & 1.26 \\
\hline $2.010^{9}$ & 0.01 & 0.46 & 0.32 & 0.67 & 1.69 \\
\hline $3.010^{9}$ & 0.01 & 0.50 & 0.33 & 0.70 & 1.74 \\
\hline $4.010^{9}$ & 0.00 & 0.54 & 0.35 & 0.73 & 1.80 \\
\hline $5.010^{9}$ & -0.01 & 0.57 & 0.36 & 0.76 & 1.84 \\
\hline $6.010^{9}$ & -0.01 & 0.60 & 0.38 & 0.79 & 1.89 \\
\hline $7.010^{9}$ & -0.02 & 0.61 & 0.39 & 0.81 & 1.92 \\
\hline $8.010^{9}$ & -0.02 & 0.63 & 0.40 & 0.82 & 1.95 \\
\hline $9.010^{9}$ & -0.02 & 0.64 & 0.41 & 0.84 & 1.98 \\
\hline $1.010^{10}$ & -0.02 & 0.65 & 0.41 & 0.85 & 2.00 \\
\hline $1.110^{10}$ & -0.02 & 0.66 & 0.42 & 0.85 & 2.01 \\
\hline $1.210^{10}$ & -0.01 & 0.67 & 0.42 & 0.87 & 2.04 \\
\hline $1.310^{10}$ & -0.01 & 0.67 & 0.42 & 0.87 & 2.05 \\
\hline $1.410^{10}$ & 0.00 & 0.67 & 0.43 & 0.88 & 2.06 \\
\hline $1.510^{10}$ & 0.01 & 0.65 & 0.42 & 0.87 & 2.05 \\
\hline $1.610^{10}$ & 0.02 & 0.66 & 0.42 & 0.88 & 2.07 \\
\hline
\end{tabular}

(1997) find slopes between 0.5 and 1.5, independent of metallicity. In this paper we use the standard Salpeter IMF with a slope of 1.35 . Variations in the slope within the mentioned range have only a small effect on the colours and indices. The low mass cut-off corresponds to the hydrogen-burning limit, which is dependent on the metallicity as shown in Chabrier \& Baraffe (1997). It ranges from $0.083 M_{\odot}$ for $[M / H]=-2.0$ to $0.075 M_{\odot}$ for $[M / H]=0$.

\subsection{Numerical method}

The SSP models presented here are single metallicity single burst models where SF occurs in one timestep, i.e. during the first $10^{7} \mathrm{yr}$. The exact duration of this burst does not affect the properties of our SSPs at ages of a few Gyr. 
Table 3. Continuation of Table 1 for $Z=0.004$

\begin{tabular}{|c|c|c|c|c|c|}
\hline time & $U-B$ & $B-V$ & $V-R$ & $V-I$ & $V-K$ \\
\hline \multicolumn{6}{|c|}{$Z=0.004$} \\
\hline $1.010^{7}$ & -0.85 & -0.13 & -0.01 & 0.01 & 0.32 \\
\hline $2.010^{7}$ & -0.72 & -0.01 & 0.12 & 0.32 & 1.26 \\
\hline $3.010^{7}$ & -0.61 & 0.04 & 0.16 & 0.41 & 1.49 \\
\hline $4.010^{7}$ & -0.50 & 0.13 & 0.21 & 0.50 & 1.64 \\
\hline $5.010^{7}$ & -0.44 & 0.13 & 0.19 & 0.47 & 1.56 \\
\hline $6.010^{7}$ & -0.42 & 0.10 & 0.16 & 0.39 & 1.38 \\
\hline $7.010^{7}$ & -0.40 & 0.09 & 0.15 & 0.36 & 1.31 \\
\hline $8.010^{7}$ & -0.38 & 0.09 & 0.14 & 0.35 & 1.26 \\
\hline $9.010^{7}$ & -0.36 & 0.09 & 0.14 & 0.35 & 1.26 \\
\hline $1.010^{8}$ & -0.33 & 0.10 & 0.14 & 0.35 & 1.26 \\
\hline $2.010^{8}$ & -0.21 & 0.14 & 0.15 & 0.35 & 1.20 \\
\hline $3.010^{8}$ & -0.13 & 0.20 & 0.18 & 0.43 & 1.32 \\
\hline $4.010^{8}$ & -0.08 & 0.23 & 0.20 & 0.46 & 1.37 \\
\hline $5.010^{8}$ & -0.02 & 0.27 & 0.21 & 0.48 & 1.39 \\
\hline $6.010^{8}$ & 0.02 & 0.28 & 0.22 & 0.48 & 1.37 \\
\hline $7.010^{8}$ & 0.06 & 0.32 & 0.23 & 0.51 & 1.42 \\
\hline $8.010^{8}$ & 0.09 & 0.35 & 0.25 & 0.54 & 1.48 \\
\hline $9.010^{8}$ & 0.11 & 0.39 & 0.27 & 0.57 & 1.54 \\
\hline $1.010^{9}$ & 0.10 & 0.41 & 0.27 & 0.59 & 1.54 \\
\hline $1.010^{9}$ & 0.10 & 0.41 & 0.27 & 0.59 & 1.54 \\
\hline $2.010^{9}$ & 0.15 & 0.64 & 0.40 & 0.82 & 2.08 \\
\hline $3.010^{9}$ & 0.18 & 0.74 & 0.45 & 0.92 & 2.27 \\
\hline $4.010^{9}$ & 0.16 & 0.75 & 0.46 & 0.94 & 2.30 \\
\hline $5.010^{9}$ & 0.17 & 0.77 & 0.47 & 0.97 & 2.36 \\
\hline $6.010^{9}$ & 0.18 & 0.79 & 0.48 & 1.00 & 2.42 \\
\hline $7.010^{9}$ & 0.20 & 0.81 & 0.50 & 1.02 & 2.46 \\
\hline $8.010^{9}$ & 0.21 & 0.82 & 0.50 & 1.03 & 2.48 \\
\hline $9.010^{9}$ & 0.22 & 0.83 & 0.51 & 1.05 & 2.52 \\
\hline $1.010^{10}$ & 0.23 & 0.84 & 0.51 & 1.06 & 2.54 \\
\hline $1.110^{10}$ & 0.24 & 0.85 & 0.52 & 1.07 & 2.56 \\
\hline $1.210^{10}$ & 0.24 & 0.85 & 0.53 & 1.08 & 2.59 \\
\hline $1.310^{10}$ & 0.26 & 0.86 & 0.53 & 1.10 & 2.64 \\
\hline $1.410^{10}$ & 0.25 & 0.86 & 0.53 & 1.10 & 2.63 \\
\hline $1.510^{10}$ & 0.25 & 0.86 & 0.53 & 1.10 & 2.64 \\
\hline $1.610^{10}$ & 0.26 & 0.86 & 0.54 & 1.11 & 2.66 \\
\hline
\end{tabular}

Theoretical stellar tracks are supplied for discrete stellar masses only. In real stellar systems, the mass distribution is expected to be continuous. Using only the discrete mass grid of the track libraries would result in severe discontinuities, since all stars of a given mass would move to the red giant branch and die at the same time. We would see many "bumps" in the luminosity and colour evolution of the stellar population. This effect is large for SSPs where all stars have about the same age. For continuous star formation rates (as in e.g. late spirals) the evolution is much smoother as expected.

To avoid the discontinuity problem we use a MonteCarlo-method to calculate the distribution of stars in the HRD at each timestep of our evolutionary synthesis model. This method was developed by Loxen (1992, 1997). For this method, no isochrones with interpolated stellar evolutionary tracks are needed. Instead, at each
Table 4. Continuation of Table 1 for $Z=0.008$

\begin{tabular}{|c|c|c|c|c|c|}
\hline time & $U-B$ & $\overline{B-V}$ & $V-R$ & $V-I$ & $V-K$ \\
\hline \multicolumn{6}{|c|}{$Z=0.008$} \\
\hline $1.010^{7}$ & -0.88 & -0.08 & 0.04 & 0.13 & 0.66 \\
\hline $2.010^{7}$ & -0.71 & -0.04 & 0.10 & 0.28 & 1.31 \\
\hline $3.010^{7}$ & -0.59 & 0.01 & 0.14 & 0.38 & 1.51 \\
\hline $4.010^{7}$ & -0.51 & 0.03 & 0.14 & 0.39 & 1.52 \\
\hline $5.010^{7}$ & -0.46 & 0.04 & 0.14 & 0.37 & 1.45 \\
\hline $6.010^{7}$ & -0.43 & 0.04 & 0.13 & 0.33 & 1.32 \\
\hline $7.010^{7}$ & -0.39 & 0.05 & 0.12 & 0.32 & 1.27 \\
\hline $8.010^{7}$ & -0.36 & 0.05 & 0.12 & 0.31 & 1.22 \\
\hline $9.010^{7}$ & -0.34 & 0.07 & 0.12 & 0.31 & 1.22 \\
\hline $1.010^{8}$ & -0.32 & 0.08 & 0.13 & 0.32 & 1.23 \\
\hline $2.010^{8}$ & -0.21 & 0.15 & 0.16 & 0.39 & 1.29 \\
\hline $3.010^{8}$ & -0.10 & 0.20 & 0.19 & 0.43 & 1.35 \\
\hline $4.010^{8}$ & -0.01 & 0.23 & 0.19 & 0.44 & 1.37 \\
\hline $5.010^{8}$ & 0.06 & 0.28 & 0.21 & 0.47 & 1.42 \\
\hline $6.010^{8}$ & 0.10 & 0.32 & 0.23 & 0.50 & 1.46 \\
\hline $7.010^{8}$ & 0.13 & 0.36 & 0.25 & 0.53 & 1.50 \\
\hline $8.010^{8}$ & 0.15 & 0.40 & 0.27 & 0.57 & 1.56 \\
\hline $9.010^{8}$ & 0.17 & 0.46 & 0.29 & 0.62 & 1.66 \\
\hline $1.010^{9}$ & 0.16 & 0.47 & 0.29 & 0.62 & 1.63 \\
\hline $1.010^{9}$ & 0.16 & 0.47 & 0.29 & 0.62 & 1.63 \\
\hline $2.010^{9}$ & 0.18 & 0.71 & 0.44 & 0.91 & 2.31 \\
\hline $3.010^{9}$ & 0.24 & 0.80 & 0.49 & 1.01 & 2.52 \\
\hline $4.010^{9}$ & 0.22 & 0.79 & 0.48 & 1.01 & 2.54 \\
\hline $5.010^{9}$ & 0.23 & 0.81 & 0.49 & 1.03 & 2.60 \\
\hline $6.010^{9}$ & 0.28 & 0.85 & 0.52 & 1.08 & 2.71 \\
\hline $7.010^{9}$ & 0.30 & 0.86 & 0.52 & 1.09 & 2.74 \\
\hline $8.010^{9}$ & 0.32 & 0.88 & 0.53 & 1.11 & 2.77 \\
\hline $9.010^{9}$ & 0.33 & 0.89 & 0.53 & 1.11 & 2.76 \\
\hline $1.010^{10}$ & 0.35 & 0.90 & 0.54 & 1.13 & 2.81 \\
\hline $1.110^{10}$ & 0.37 & 0.91 & 0.55 & 1.15 & 2.84 \\
\hline $1.210^{10}$ & 0.39 & 0.92 & 0.56 & 1.17 & 2.89 \\
\hline $1.310^{10}$ & 0.41 & 0.93 & 0.56 & 1.16 & 2.87 \\
\hline $1.410^{10}$ & 0.42 & 0.94 & 0.56 & 1.19 & 2.94 \\
\hline $1.510^{10}$ & 0.42 & 0.93 & 0.56 & 1.19 & 2.96 \\
\hline $1.610^{10}$ & 0.42 & 0.94 & 0.57 & 1.20 & 2.94 \\
\hline
\end{tabular}

timestep a large grid in stellar masses and ages is created. The grid is created randomly, hence the Monte-Carlo designation. Each cell in this grid has a size $\Delta m \cdot \Delta t$ in the 2 -dimensional mass - time space. The grid ranges in mass from the lower mass to upper mass cutoff. In the case of an SSP the grid ranges in time from zero to the end of the burst, while for a continous SFR this would be from zero to the model age. Each cell represents a pseudo star, which is weighted with the value of the initial mass function (IMF) and the value of the star formation rate (SFR) at the position of the cell. This weight is given by

$w=\Delta m \cdot \phi(m) \cdot \Delta t \cdot \psi(t)$,

where $\phi(m)$ is the value of the IMF at the mass of the pseudo star and $\psi(t)$ is the star formation rate at the time the pseudo star is born. In the case of an SSP, the function $\psi(t)$ is constant over the time of the burst and then equal to zero. The unit of the value $w$ is number of 
Table 5. Continuation of Table 1 for $Z=0.02$

\begin{tabular}{|c|c|c|c|c|c|}
\hline time & $U-B$ & $B-V$ & $V-R$ & $V-I$ & $\overline{V-K}$ \\
\hline \multicolumn{6}{|c|}{$Z=0.02$} \\
\hline $1.010^{7}$ & -0.93 & -0.14 & 0.02 & 0.12 & 0.95 \\
\hline $2.010^{7}$ & -0.70 & 0.05 & 0.22 & 0.62 & 2.23 \\
\hline $3.010^{7}$ & -0.64 & 0.04 & 0.20 & 0.58 & 2.16 \\
\hline $4.010^{7}$ & -0.56 & 0.04 & 0.18 & 0.49 & 1.90 \\
\hline $5.010^{7}$ & -0.50 & 0.07 & 0.18 & 0.47 & 1.78 \\
\hline $6.010^{7}$ & -0.46 & 0.07 & 0.17 & 0.42 & 1.61 \\
\hline $7.010^{7}$ & -0.42 & 0.09 & 0.17 & 0.42 & 1.59 \\
\hline $8.010^{7}$ & -0.40 & 0.09 & 0.16 & 0.40 & 1.54 \\
\hline $9.010^{7}$ & -0.37 & 0.09 & 0.16 & 0.40 & 1.53 \\
\hline $1.010^{8}$ & -0.34 & 0.10 & 0.16 & 0.40 & 1.53 \\
\hline $2.010^{8}$ & -0.15 & 0.14 & 0.15 & 0.37 & 1.41 \\
\hline $3.010^{8}$ & -0.01 & 0.20 & 0.18 & 0.41 & 1.45 \\
\hline $4.010^{8}$ & 0.10 & 0.27 & 0.20 & 0.46 & 1.50 \\
\hline $5.010^{8}$ & 0.16 & 0.32 & 0.23 & 0.51 & 1.55 \\
\hline $6.010^{8}$ & 0.20 & 0.40 & 0.26 & 0.56 & 1.63 \\
\hline $7.010^{8}$ & 0.21 & 0.46 & 0.29 & 0.60 & 1.69 \\
\hline $8.010^{8}$ & 0.21 & 0.51 & 0.31 & 0.64 & 1.76 \\
\hline $9.010^{8}$ & 0.22 & 0.56 & 0.33 & 0.68 & 1.82 \\
\hline $1.010^{9}$ & 0.18 & 0.56 & 0.33 & 0.68 & 1.80 \\
\hline $1.010^{9}$ & 0.18 & 0.56 & 0.33 & 0.68 & 1.80 \\
\hline $2.010^{9}$ & 0.26 & 0.77 & 0.47 & 0.98 & 2.58 \\
\hline $3.010^{9}$ & 0.37 & 0.87 & 0.52 & 1.09 & 2.85 \\
\hline $4.010^{9}$ & 0.40 & 0.90 & 0.54 & 1.11 & 2.93 \\
\hline $5.010^{9}$ & 0.43 & 0.92 & 0.55 & 1.14 & 2.99 \\
\hline $6.010^{9}$ & 0.46 & 0.94 & 0.56 & 1.16 & 3.05 \\
\hline $7.010^{9}$ & 0.47 & 0.94 & 0.56 & 1.17 & 3.06 \\
\hline $8.010^{9}$ & 0.50 & 0.95 & 0.57 & 1.18 & 3.08 \\
\hline $9.010^{9}$ & 0.53 & 0.97 & 0.58 & 1.21 & 3.14 \\
\hline $1.010^{10}$ & 0.56 & 0.99 & 0.58 & 1.22 & 3.18 \\
\hline $1.110^{10}$ & 0.59 & 1.00 & 0.59 & 1.24 & 3.25 \\
\hline $1.210^{10}$ & 0.61 & 1.01 & 0.60 & 1.25 & 3.23 \\
\hline $1.310^{10}$ & 0.64 & 1.02 & 0.60 & 1.25 & 3.21 \\
\hline $1.410^{10}$ & 0.66 & 1.03 & 0.61 & 1.27 & 3.29 \\
\hline $1.510^{10}$ & 0.68 & 1.04 & 0.61 & 1.28 & 3.27 \\
\hline $1.610^{10}$ & 0.70 & 1.05 & 0.62 & 1.29 & 3.34 \\
\hline
\end{tabular}

stars, although it can be fractional. For each of these cells, the lifetime of the pseudo star is determined by interpolation between the nearest two stellar tracks supplied in the library. Then the timesteps of the tracks are stretched according to this new lifetime. The cell is then split in two parts with relative weights given by

$w_{1}=\frac{\log \left(m_{2}\right)-\log (m)}{\log \left(m_{2}\right)-\log \left(m_{1}\right)} \cdot w ; \quad w_{2}=w-w_{1}$,

where $m$ is the mass value of the cell, $m_{1}$ is the mass of the track with the next lower mass and $m_{2}$ is the mass of the track with the next higher mass.

The only interpolation that is done is to determine the life times and hence the duration of the individual states. No interpolation is done for the luminosities or the effective temperatures which would require a precise definition of equivalent evolutionary stages. In this way, no artificial tracks are created. Especially in mass ranges were there is
Table 6. Continuation of Table 1 for $Z=0.05$

\begin{tabular}{|c|c|c|c|c|c|}
\hline time & $U-B$ & $\overline{B-V}$ & $V-R$ & $\overline{V-I}$ & $V-K$ \\
\hline \multicolumn{6}{|c|}{$Z=0.05$} \\
\hline $1.010^{7}$ & -0.95 & -0.18 & -0.01 & 0.18 & 1.74 \\
\hline $2.010^{7}$ & -0.77 & -0.14 & 0.04 & 0.52 & 3.07 \\
\hline $3.010^{7}$ & -0.65 & -0.09 & 0.07 & 0.49 & 2.64 \\
\hline $4.010^{7}$ & -0.56 & -0.04 & 0.11 & 0.50 & 2.38 \\
\hline $5.010^{7}$ & -0.50 & -0.01 & 0.13 & 0.46 & 2.13 \\
\hline $6.010^{7}$ & -0.45 & 0.01 & 0.14 & 0.44 & 1.99 \\
\hline $7.010^{7}$ & -0.40 & 0.02 & 0.14 & 0.43 & 1.93 \\
\hline $8.010^{7}$ & -0.36 & 0.04 & 0.15 & 0.43 & 1.89 \\
\hline $9.010^{7}$ & -0.33 & 0.05 & 0.15 & 0.41 & 1.81 \\
\hline $1.010^{8}$ & -0.30 & 0.06 & 0.15 & 0.40 & 1.75 \\
\hline $2.010^{8}$ & -0.07 & 0.15 & 0.18 & 0.43 & 1.69 \\
\hline $3.010^{8}$ & 0.10 & 0.26 & 0.23 & 0.51 & 1.82 \\
\hline $4.010^{8}$ & 0.18 & 0.34 & 0.26 & 0.56 & 1.87 \\
\hline $5.010^{8}$ & 0.23 & 0.44 & 0.30 & 0.63 & 1.99 \\
\hline $6.010^{8}$ & 0.25 & 0.51 & 0.33 & 0.68 & 2.06 \\
\hline $7.010^{8}$ & 0.27 & 0.58 & 0.36 & 0.74 & 2.16 \\
\hline $8.010^{8}$ & 0.28 & 0.64 & 0.39 & 0.79 & 2.25 \\
\hline $9.010^{8}$ & 0.29 & 0.70 & 0.42 & 0.83 & 2.33 \\
\hline $1.010^{9}$ & 0.36 & 0.79 & 0.46 & 0.91 & 2.47 \\
\hline $1.010^{9}$ & 0.36 & 0.79 & 0.46 & 0.91 & 2.47 \\
\hline $2.010^{9}$ & 0.45 & 0.89 & 0.54 & 1.11 & 3.04 \\
\hline $3.010^{9}$ & 0.53 & 0.94 & 0.57 & 1.17 & 3.21 \\
\hline $4.010^{9}$ & 0.67 & 1.02 & 0.62 & 1.26 & 3.43 \\
\hline $5.010^{9}$ & 0.69 & 1.03 & 0.62 & 1.26 & 3.41 \\
\hline $6.010^{9}$ & 0.76 & 1.06 & 0.63 & 1.29 & 3.47 \\
\hline $7.010^{9}$ & 0.81 & 1.08 & 0.65 & 1.32 & 3.53 \\
\hline $8.010^{9}$ & 0.83 & 1.08 & 0.65 & 1.32 & 3.55 \\
\hline $9.010^{9}$ & 0.87 & 1.10 & 0.66 & 1.34 & 3.58 \\
\hline $1.010^{10}$ & 0.89 & 1.11 & 0.66 & 1.36 & 3.61 \\
\hline $1.110^{10}$ & 0.91 & 1.12 & 0.67 & 1.36 & 3.66 \\
\hline $1.210^{10}$ & 0.92 & 1.12 & 0.67 & 1.36 & 3.62 \\
\hline $1.310^{10}$ & 0.95 & 1.13 & 0.67 & 1.37 & 3.63 \\
\hline $1.410^{10}$ & 0.97 & 1.14 & 0.67 & 1.38 & 3.63 \\
\hline $1.510^{10}$ & 0.98 & 1.14 & 0.68 & 1.39 & 3.67 \\
\hline $1.610^{10}$ & 1.00 & 1.15 & 0.68 & 1.39 & 3.69 \\
\hline
\end{tabular}

a strong dependance of the stellar evolution on the mass this is important.

The cells in the grid do not represent individual stars since their weights are not necessarily unity. The weights $w$ are added in a book-keeping list for the individual states in the tracks of the input library to get weights $W_{i, j}$. Thereafter, the luminosities of all states on all tracks are summed up, each weighted with the calculated weights:

$L_{\text {total }}=\sum_{i, j} W_{i, j} \cdot L_{i, j}$

where $L_{\text {total }}$ is the luminosity in some band, $W_{i, j}$ are the assigned weights and $L_{i, j}$ are the luminosities of a theoretical star from the library at $i$ th mass and $j$ th state.

Due to the randomly spaced grid, there is a noise on the results, but the finer the grid (i.e. the more cells in the grid), the better is the signal-to-noise ratio. For the models in this paper, we use 200000 masses distributed evenly 
in log mass and 1000 ages distributed evenly, which make up a grid of $210^{8}$ cells. Any further increase of the size of the grid does not give significantly better results. For more continuous SFRs, the grid can be coarser. Trivially the grid extends in time such that all stars are born in the burst interval, i.e. in the first $10^{7}$ years. This implies that each $\Delta t$ from Eq. (2) is equal to around $10^{4}$ years.

For the same input physics data set, the Monte Carlo method has been tested in detail against the standard synthesis model for SSPs (Fritze - v. Alvensleben 1995). Results for solar metallicity are compared to those of various evolutionary synthesis codes including the isochrone synthesis code of Bruzual \& Charlot in Sect. 4.3.

On our Linux Pentium II $300 \mathrm{MHz}$ work stations a single run with a timestep of one Gyr needs a few minutes of cpu time for the evolution of an SSP over a Hubble time.

This method has many advantages. Models at any time can be calculated without the need of any interpolation between stellar evolutionary tracks avoiding the problem of defining equivalent evolutionary stages, i.e. only the time intervals of the individual tracks are interpolated (but not the temperatures or gravities and therefore the colours). It is also possible to use an arbitrary star formation history. In the future, this code will be extended to be able to work with arbitrary metallicities not covered by the stellar tracks available. Since this is an evolutionary synthesis code rather than a population synthesis code, it can also be extended to calculate the chemical enrichment of galaxies.

\section{Variation of input parameters}

\subsection{Stellar mass loss}

The mass loss of stars, especially for low mass stars, is still not understood very well. A formulae often used to describe mass loss is Reimers' law:

$\dot{M}=1.2710^{-5} \cdot \eta M^{-1} L^{1.5} T_{\mathrm{eff}}^{-2}$,

where the parameter $\eta$ is not known a priori and is probably a function of metallicity.

On the horizontal branch, stars tend to be hotter the lower their mass, thus a larger value of $\eta$ makes the HB population bluer.

In this work we adopt for $\eta$ a value of 0.35 independent of metallicity for lack of better knowledge. For a careful study of the effects of changing $\eta$ e.g. on the HB morphology see Buzzoni et al. (1989). Covino et al. (1994) present an extensive study of the effect of different HB morphologies of theoretical isochrones on the integrated colours.

We found that there is no signifcant effect of the HB morphology of observed Galactic globular clusters on the optical colours.

\subsection{Non-solar abundance ratios}

There are many observations showing that the $\alpha$ element-to-iron ratio is larger than solar for stars with low metallicities in the Milky Way (e.g. Furmann et al. 1995). Nevertheless, most stellar evolutionary track calculations for sub-solar metallicities use solar abundance ratios. Salaris et al. (1993) show that the effect of different $\alpha$-to-Fe element ratios on the stellar evolution can be accounted for by appropriately changing the conversion of global metallicity $Z$ to $[\mathrm{Fe} / \mathrm{H}]$. Despite the observed $\alpha$-enhancement for metal poor stars in the MW, no correction is applied since we checked that a correction did not give better fits to the observations. In this paper, $\log \left(\frac{Z}{Z_{\odot}}\right)(=:[M / H])$ was assumed to be equal to $[\mathrm{Fe} / \mathrm{H}]$.

For absorption indices, any correction for $\alpha$ - enhancement would be inappropriate for our models since we are using the index calibrations from Worthey, which depend only on the metallicity and not explicitily on $[\alpha / \mathrm{Fe}]$ - element ratios. Since Worthey's index calibrations are determined from Galactic stars, the dependence of $\alpha$ - enhancement on metallicity is so implicitly included.

Somewhat surprisingly we found no previous study that has examined the effect of $[\alpha / \mathrm{Fe}]$ on the colours at fixed temperature. An analysis by Kurth (1998) shows that at least for solar type stars, the colour is only dependent on $[\mathrm{Fe} / \mathrm{H}]$ and not on $[\mathrm{Mg} / \mathrm{Fe}]$.

There may still be an effect of non-solar abundance ratios on the morphology of the horizontal branch, but since our models agree with the observations no further investigation in this direction is attempted at the present stage.

\section{Results}

\subsection{Colours}

Figures 1 and 2 show the evolution of $B-V$ and $V-I$ versus time of our SSPs on a logaritmic time scale for all metallicities. After a few Gyrs the changes in both colours become very slow, and the metallicity dependence becomes more important. Tables 1 through 3 give the time evolution of all our broad band colours from $U$ to $K$ for 6 metallicities from $Z=0.0001$ to $Z=0.05$ for a wide range of ages.

In Figs. 3 and 4 the colours $B-V$ and $V-I$ are shown as a function of metallicity for the models at evolutionary ages of 10, 12 and 15 Gyr together with colors of dereddened GCs from the McMaster catalogue (Harris 1996). In general, we see a good agreement between the models and the observed clusters. The large spread in colour in the observed clusters probably arises from observational errors.

It can also be seen in both models and observations, that for very low metallicities the colour-metallicity relation cannot be expressed by a simple linear function. For 
Table 7. Indices. Time is in years. See Worthey (1994) for index definitions

\begin{tabular}{|c|c|c|c|c|c|c|c|c|c|}
\hline time & $\mathrm{H}_{\beta}$ & $\mathrm{Mg}_{1}$ & $\mathrm{Mg}_{2}$ & $\mathrm{Mg}_{b}$ & Fe5270 & Fe5335 & $\mathrm{NaD}$ & $\mathrm{TiO}_{1}$ & $\mathrm{TiO}_{2}$ \\
\hline \multicolumn{10}{|c|}{$\overline{Z Z}=0.0001$} \\
\hline $5.010^{8}$ & 6.50 & 0.020 & -0.003 & 0.11 & -1.31 & 0.20 & 2.12 & 0.013 & -0.006 \\
\hline $1.010^{9}$ & 6.38 & 0.014 & 0.002 & 0.30 & -0.99 & 0.31 & 2.41 & 0.013 & -0.005 \\
\hline $2.010^{9}$ & 4.94 & 0.003 & 0.009 & 0.31 & -0.49 & 0.38 & 2.36 & 0.013 & -0.003 \\
\hline $3.010^{9}$ & 4.64 & 0.001 & 0.013 & 0.44 & -0.36 & 0.44 & 2.44 & 0.013 & -0.003 \\
\hline $4.010^{9}$ & 4.21 & 0.000 & 0.015 & 0.49 & -0.23 & 0.48 & 2.48 & 0.013 & -0.003 \\
\hline $5.010^{9}$ & 3.89 & -0.000 & 0.017 & 0.52 & -0.16 & 0.51 & 2.50 & 0.012 & -0.003 \\
\hline $6.010^{9}$ & 3.57 & 0.000 & 0.019 & 0.53 & -0.09 & 0.53 & 2.50 & 0.012 & -0.004 \\
\hline $7.010^{9}$ & 3.33 & 0.001 & 0.019 & 0.51 & -0.03 & 0.56 & 2.54 & 0.012 & -0.004 \\
\hline $8.010^{9}$ & 3.13 & 0.001 & 0.018 & 0.47 & 0.02 & 0.58 & 2.57 & 0.012 & -0.004 \\
\hline $9.010^{9}$ & 3.01 & 0.002 & 0.018 & 0.43 & 0.05 & 0.61 & 2.61 & 0.011 & -0.005 \\
\hline $1.010^{10}$ & 2.95 & 0.003 & 0.018 & 0.44 & 0.07 & 0.62 & 2.62 & 0.011 & -0.005 \\
\hline $1.110^{10}$ & 2.90 & 0.004 & 0.019 & 0.46 & 0.09 & 0.63 & 2.64 & 0.011 & -0.005 \\
\hline $1.210^{10}$ & 2.90 & 0.004 & 0.020 & 0.48 & 0.10 & 0.64 & 2.65 & 0.011 & -0.005 \\
\hline $1.310^{10}$ & 2.92 & 0.005 & 0.021 & 0.50 & 0.11 & 0.65 & 2.64 & 0.011 & -0.006 \\
\hline $1.410^{10}$ & 2.94 & 0.007 & 0.023 & 0.53 & 0.11 & 0.66 & 2.68 & 0.010 & -0.006 \\
\hline $1.510^{10}$ & 2.92 & 0.008 & 0.024 & 0.54 & 0.12 & 0.67 & 2.69 & 0.010 & -0.006 \\
\hline $1.610^{10}$ & 2.93 & 0.010 & 0.026 & 0.57 & 0.12 & 0.69 & 2.74 & 0.010 & -0.007 \\
\hline \multicolumn{10}{|c|}{$Z=0.0004$} \\
\hline $5.010^{8}$ & 6.14 & 0.010 & 0.014 & 0.35 & -0.60 & 0.02 & 1.85 & 0.013 & -0.005 \\
\hline $1.010^{9}$ & 5.33 & 0.004 & 0.023 & 0.35 & -0.18 & 0.22 & 2.15 & 0.013 & -0.003 \\
\hline $2.010^{9}$ & 4.43 & 0.003 & 0.026 & 0.68 & 0.30 & 0.43 & 2.05 & 0.013 & -0.001 \\
\hline $3.010^{9}$ & 4.16 & 0.003 & 0.028 & 0.71 & 0.37 & 0.46 & 2.04 & 0.013 & -0.001 \\
\hline $4.010^{9}$ & 3.79 & 0.003 & 0.031 & 0.76 & 0.47 & 0.52 & 2.08 & 0.013 & -0.001 \\
\hline $5.010^{9}$ & 3.47 & 0.003 & 0.033 & 0.80 & 0.54 & 0.56 & 2.10 & 0.013 & -0.001 \\
\hline $6.010^{9}$ & 3.17 & 0.004 & 0.038 & 0.88 & 0.62 & 0.61 & 2.14 & 0.013 & -0.001 \\
\hline $7.010^{9}$ & 2.97 & 0.006 & 0.042 & 0.94 & 0.67 & 0.65 & 2.18 & 0.012 & -0.002 \\
\hline $8.010^{9}$ & 2.80 & 0.006 & 0.043 & 0.95 & 0.72 & 0.68 & 2.21 & 0.012 & -0.002 \\
\hline $9.010^{9}$ & 2.66 & 0.007 & 0.044 & 0.93 & 0.76 & 0.70 & 2.23 & 0.012 & -0.002 \\
\hline $1.010^{10}$ & 2.58 & 0.008 & 0.044 & 0.90 & 0.78 & 0.72 & 2.28 & 0.012 & -0.002 \\
\hline $1.110^{10}$ & 2.50 & 0.009 & 0.044 & 0.88 & 0.80 & 0.74 & 2.31 & 0.012 & -0.002 \\
\hline $1.210^{10}$ & 2.42 & 0.009 & 0.044 & 0.85 & 0.83 & 0.76 & 2.31 & 0.011 & -0.002 \\
\hline $1.310^{10}$ & 2.38 & 0.010 & 0.045 & 0.84 & 0.85 & 0.77 & 2.32 & 0.011 & -0.002 \\
\hline $1.410^{10}$ & 2.42 & 0.011 & 0.046 & 0.85 & 0.85 & 0.78 & 2.32 & 0.011 & -0.003 \\
\hline $1.510^{10}$ & 2.59 & 0.012 & 0.048 & 0.89 & 0.81 & 0.77 & 2.33 & 0.011 & -0.003 \\
\hline $1.610^{10}$ & 2.58 & 0.013 & 0.049 & 0.91 & 0.83 & 0.78 & 2.33 & 0.011 & -0.003 \\
\hline \multicolumn{10}{|c|}{$Z=0.004$} \\
\hline $5.010^{8}$ & 6.10 & 0.016 & 0.048 & 0.85 & 0.56 & 0.39 & 1.50 & 0.016 & 0.003 \\
\hline $1.010^{9}$ & 5.31 & 0.017 & 0.061 & 1.14 & 0.96 & 0.70 & 1.80 & 0.014 & 0.001 \\
\hline $2.010^{9}$ & 3.66 & 0.030 & 0.095 & 1.64 & 1.53 & 1.15 & 1.72 & 0.020 & 0.016 \\
\hline $3.010^{9}$ & 2.79 & 0.037 & 0.111 & 1.86 & 1.78 & 1.35 & 1.72 & 0.024 & 0.024 \\
\hline $4.010^{9}$ & 2.63 & 0.038 & 0.112 & 1.84 & 1.80 & 1.38 & 1.81 & 0.025 & 0.026 \\
\hline $5.010^{9}$ & 2.42 & 0.041 & 0.117 & 1.92 & 1.86 & 1.45 & 1.91 & 0.026 & 0.029 \\
\hline $6.010^{9}$ & 2.26 & 0.044 & 0.124 & 2.02 & 1.92 & 1.51 & 1.99 & 0.028 & 0.032 \\
\hline $7.010^{9}$ & 2.12 & 0.047 & 0.129 & 2.10 & 1.98 & 1.56 & 2.04 & 0.029 & 0.034 \\
\hline $8.010^{9}$ & 2.03 & 0.049 & 0.133 & 2.18 & 2.01 & 1.60 & 2.11 & 0.029 & 0.034 \\
\hline $9.010^{9}$ & 1.97 & 0.052 & 0.138 & 2.26 & 2.04 & 1.64 & 2.20 & 0.030 & 0.036 \\
\hline $1.010^{10}$ & 1.90 & 0.054 & 0.141 & 2.32 & 2.08 & 1.68 & 2.29 & 0.030 & 0.036 \\
\hline $1.110^{10}$ & 1.86 & 0.057 & 0.146 & 2.39 & 2.11 & 1.72 & 2.38 & 0.030 & 0.037 \\
\hline $1.210^{10}$ & 1.82 & 0.059 & 0.150 & 2.45 & 2.13 & 1.76 & 2.48 & 0.031 & 0.039 \\
\hline $1.310^{10}$ & 1.78 & 0.062 & 0.155 & 2.52 & 2.16 & 1.80 & 2.57 & 0.033 & 0.043 \\
\hline $1.410^{10}$ & 1.77 & 0.062 & 0.156 & 2.54 & 2.16 & 1.80 & 2.59 & 0.032 & 0.041 \\
\hline $1.510^{10}$ & 1.77 & 0.062 & 0.158 & 2.55 & 2.16 & 1.80 & 2.60 & 0.033 & 0.042 \\
\hline $1.610^{10}$ & 1.76 & 0.063 & 0.162 & 2.57 & 2.17 & 1.82 & 2.61 & 0.033 & 0.042 \\
\hline
\end{tabular}


Table 8. Continuation of Table 7

\begin{tabular}{|c|c|c|c|c|c|c|c|c|c|}
\hline time & $\mathrm{H}_{\beta}$ & $\mathrm{Mg}_{1}$ & $\mathrm{Mg}_{2}$ & $\mathrm{Mg}_{b}$ & Fe5270 & Fe5335 & $\mathrm{NaD}$ & $\mathrm{TiO}_{1}$ & $\mathrm{TiO}_{2}$ \\
\hline \multicolumn{10}{|c|}{$Z=0.008$} \\
\hline $5.010^{8}$ & 6.41 & 0.018 & 0.057 & 0.98 & 0.84 & 0.59 & 1.39 & 0.016 & 0.004 \\
\hline $1.010^{9}$ & 5.05 & 0.022 & 0.076 & 1.38 & 1.31 & 1.00 & 1.69 & 0.016 & 0.005 \\
\hline $2.010^{9}$ & 3.16 & 0.044 & 0.128 & 2.01 & 1.95 & 1.57 & 1.94 & 0.030 & 0.035 \\
\hline $3.010^{9}$ & 2.50 & 0.055 & 0.147 & 2.32 & 2.19 & 1.81 & 2.13 & 0.034 & 0.044 \\
\hline $4.010^{9}$ & 2.41 & 0.055 & 0.149 & 2.38 & 2.17 & 1.81 & 2.23 & 0.034 & 0.043 \\
\hline $5.010^{9}$ & 2.25 & 0.058 & 0.157 & 2.52 & 2.23 & 1.88 & 2.32 & 0.035 & 0.046 \\
\hline $6.010^{9}$ & 2.05 & 0.065 & 0.170 & 2.68 & 2.34 & 1.99 & 2.41 & 0.038 & 0.051 \\
\hline $7.010^{9}$ & 1.97 & 0.067 & 0.175 & 2.76 & 2.38 & 2.03 & 2.48 & 0.038 & 0.052 \\
\hline $8.010^{9}$ & 1.89 & 0.070 & 0.180 & 2.84 & 2.42 & 2.08 & 2.55 & 0.039 & 0.053 \\
\hline $9.010^{9}$ & 1.82 & 0.072 & 0.184 & 2.90 & 2.45 & 2.11 & 2.60 & 0.038 & 0.052 \\
\hline $1.010^{10}$ & 1.76 & 0.075 & 0.189 & 2.96 & 2.49 & 2.15 & 2.66 & 0.039 & 0.054 \\
\hline $1.110^{10}$ & 1.70 & 0.077 & 0.194 & 3.02 & 2.53 & 2.19 & 2.71 & 0.040 & 0.055 \\
\hline $1.210^{10}$ & 1.64 & 0.080 & 0.198 & 3.07 & 2.56 & 2.23 & 2.77 & 0.041 & 0.057 \\
\hline $1.310^{10}$ & 1.59 & 0.082 & 0.200 & 3.09 & 2.58 & 2.25 & 2.80 & 0.039 & 0.054 \\
\hline $1.410^{10}$ & 1.56 & 0.084 & 0.203 & 3.13 & 2.60 & 2.27 & 2.86 & 0.042 & 0.059 \\
\hline $1.510^{10}$ & 1.54 & 0.085 & 0.206 & 3.16 & 2.61 & 2.29 & 2.93 & 0.042 & 0.059 \\
\hline $1.610^{10}$ & 1.52 & 0.087 & 0.208 & 3.19 & 2.62 & 2.32 & 2.99 & 0.042 & 0.059 \\
\hline \multicolumn{10}{|c|}{$Z=0.02$} \\
\hline $5.010^{8}$ & 6.41 & 0.018 & 0.079 & 1.22 & 1.28 & 1.00 & 1.33 & 0.020 & 0.010 \\
\hline $1.010^{9}$ & 4.44 & 0.027 & 0.108 & 1.78 & 1.80 & 1.51 & 1.70 & 0.021 & 0.014 \\
\hline $2.010^{9}$ & 2.78 & 0.066 & 0.172 & 2.58 & 2.43 & 2.18 & 2.48 & 0.036 & 0.049 \\
\hline $3.010^{9}$ & 2.20 & 0.084 & 0.204 & 3.10 & 2.72 & 2.47 & 2.80 & 0.039 & 0.056 \\
\hline $4.010^{9}$ & 2.02 & 0.090 & 0.216 & 3.28 & 2.80 & 2.56 & 2.93 & 0.040 & 0.058 \\
\hline $5.010^{9}$ & 1.90 & 0.095 & 0.225 & 3.41 & 2.86 & 2.63 & 3.04 & 0.040 & 0.059 \\
\hline $6.010^{9}$ & 1.81 & 0.100 & 0.233 & 3.51 & 2.91 & 2.69 & 3.15 & 0.042 & 0.062 \\
\hline $7.010^{9}$ & 1.74 & 0.102 & 0.237 & 3.58 & 2.94 & 2.73 & 3.23 & 0.042 & 0.062 \\
\hline $8.010^{9}$ & 1.68 & 0.104 & 0.242 & 3.65 & 2.98 & 2.77 & 3.30 & 0.042 & 0.063 \\
\hline $9.010^{9}$ & 1.60 & 0.109 & 0.250 & 3.73 & 3.04 & 2.84 & 3.39 & 0.043 & 0.065 \\
\hline $1.010^{10}$ & 1.54 & 0.113 & 0.256 & 3.79 & 3.08 & 2.89 & 3.48 & 0.044 & 0.066 \\
\hline $1.110^{10}$ & 1.49 & 0.116 & 0.261 & 3.85 & 3.12 & 2.93 & 3.55 & 0.045 & 0.069 \\
\hline $1.210^{10}$ & 1.44 & 0.118 & 0.265 & 3.89 & 3.15 & 2.96 & 3.61 & 0.045 & 0.069 \\
\hline $1.310^{10}$ & 1.39 & 0.121 & 0.269 & 3.93 & 3.18 & 3.00 & 3.67 & 0.045 & 0.069 \\
\hline $1.410^{10}$ & 1.35 & 0.123 & 0.273 & 3.97 & 3.21 & 3.03 & 3.73 & 0.046 & 0.072 \\
\hline $1.510^{10}$ & 1.32 & 0.125 & 0.276 & 4.01 & 3.23 & 3.06 & 3.78 & 0.046 & 0.072 \\
\hline $1.610^{10}$ & 1.29 & 0.127 & 0.278 & 4.03 & 3.25 & 3.08 & 3.83 & 0.047 & 0.073 \\
\hline \multicolumn{10}{|c|}{$Z=0.05$} \\
\hline $5.010^{8}$ & 5.79 & 0.035 & 0.116 & 1.70 & 1.89 & 1.74 & 1.87 & 0.023 & 0.021 \\
\hline $1.010^{9}$ & 3.15 & 0.079 & 0.194 & 2.84 & 2.78 & 2.66 & 2.89 & 0.026 & 0.032 \\
\hline $2.010^{9}$ & 2.29 & 0.103 & 0.235 & 3.46 & 3.04 & 3.01 & 3.29 & 0.041 & 0.065 \\
\hline $3.010^{9}$ & 1.97 & 0.114 & 0.256 & 3.81 & 3.18 & 3.18 & 3.52 & 0.043 & 0.070 \\
\hline $4.010^{9}$ & 1.66 & 0.140 & 0.294 & 4.22 & 3.45 & 3.48 & 4.01 & 0.049 & 0.084 \\
\hline $5.010^{9}$ & 1.59 & 0.140 & 0.297 & 4.30 & 3.46 & 3.50 & 4.06 & 0.048 & 0.082 \\
\hline $6.010^{9}$ & 1.46 & 0.149 & 0.311 & 4.44 & 3.55 & 3.61 & 4.25 & 0.050 & 0.086 \\
\hline $7.010^{9}$ & 1.38 & 0.156 & 0.322 & 4.54 & 3.62 & 3.69 & 4.41 & 0.052 & 0.090 \\
\hline $8.010^{9}$ & 1.33 & 0.159 & 0.327 & 4.60 & 3.66 & 3.73 & 4.52 & 0.052 & 0.090 \\
\hline $9.010^{9}$ & 1.27 & 0.164 & 0.335 & 4.68 & 3.71 & 3.79 & 4.66 & 0.053 & 0.093 \\
\hline $1.010^{10}$ & 1.23 & 0.168 & 0.342 & 4.74 & 3.76 & 3.84 & 4.79 & 0.054 & 0.096 \\
\hline $1.110^{10}$ & 1.20 & 0.170 & 0.345 & 4.77 & 3.79 & 3.88 & 4.89 & 0.055 & 0.096 \\
\hline $1.210^{10}$ & 1.17 & 0.172 & 0.349 & 4.81 & 3.82 & 3.91 & 5.00 & 0.055 & 0.095 \\
\hline $1.310^{10}$ & 1.13 & 0.175 & 0.355 & 4.86 & 3.86 & 3.95 & 5.11 & 0.055 & 0.096 \\
\hline $1.410^{10}$ & 1.10 & 0.178 & 0.359 & 4.89 & 3.89 & 3.99 & 5.20 & 0.055 & 0.096 \\
\hline $1.510^{10}$ & 1.07 & 0.180 & 0.362 & 4.92 & 3.91 & 4.02 & 5.28 & 0.056 & 0.098 \\
\hline $1.610^{10}$ & 1.05 & 0.182 & 0.365 & 4.94 & 3.93 & 4.04 & 5.34 & 0.056 & 0.098 \\
\hline
\end{tabular}


$[M / H] \lesssim-1.7$, the relation becomes significantly flatter. The flattening is particularly pronounced in $V-I$. The models also show that the $(V-I)$-metallicity relation steepens for $[M / H]>0$. Very often the metallicity is calculated for globular cluster systems in other galaxies from $V-I$ using a simple linear regression line calculated from Galactic GCs. The models show that this approach is dangerous for metallicities higher than those of Galactic GCs. A quadratic or higher order fit would not improve things if calculated for low metallicities and applied to higher metallicities as it would include further uncertainties. Therefore a theoretical calibration like the one provided here is to be preferred.

\subsection{Indices}

In Figs. 5 and 6 we plot against metallicity the indices $\mathrm{Mg}_{2}$ and Fe5270 from our models and those of Galactic clusters from Burstein (1984). The agreement of the models with the observations is very good over the metalicity range covered by the data. Model calibrations of both $\mathrm{Mg}_{2}$ and Fe5270 as functions of metallicity are almost independent of age for ages close to a Hubble time.

Figure 7 shows the $\mathrm{H}_{\beta}$ index against the $\mathrm{Mg}_{2}$ for Galactic GCs and clusters from M 31. As can be seen in the figure the $H_{\beta}$ index is higher for higher metallicity for the M 31 GCs than for the Milky Way clusters. This was already noted by Burstein (1984) though the reason for this discrepancy is still unknown. The models fall right between the two groups. It can also be seen that the $\mathrm{H}_{\beta}$ to $\mathrm{Mg}_{2}$ relation is dependent largely on age. If it were not for the large spread present in the $\mathrm{H}_{\beta}$ observations this index would be a good tool to disentangle age from metallicity.

The Fe5335 and $\mathrm{TiO}_{1}$ are plotted against $\mathrm{Mg}_{2}$ for observations from Burstein in Figs. 8 and 9 respectively. Both the relations between the $\mathrm{Fe} 5335$ and $\mathrm{TiO}_{1}$ indices and $\mathrm{Mg}_{2}$ are virtually independent of model age for ages close to a Hubble time.

In Fig. 10 we show the model index $\mathrm{Mg}_{2}$ against metallicity for a wide range of ages from 0.5 to 12 Gyrs.

\subsection{Comparison with other authors}

We compared our results with those of Bruzual \& Charlot (BC96) (1996), Vazdekis et al. (1996), Worthey (1994), Tantalo et al. (1996) (for $B-V$ ) and Tantalo et al. (1998) (for $\mathrm{Mg}_{2}$ ). With the exception of the models from Worthey, all models are based on the Padua tracks.

Our $B-V$ - colours are very close to those of the BC96 models. This is surprising since they also calibrated their colours with the library from Lejeune. The models from Vazdekis et al. are bluer at all times. They use a different empirical calibration. The $B-V$ - colours for all models seem to converge at high ages.
The calibration of the indices are from Worthey (1994) for all models with the exception of the models from Tantalo et al. (1998), who use the empirical calibrations from Borges et al. (1995) for $\mathrm{Mg}_{2}$ which depend on the $[\mathrm{Mg} / \mathrm{Fe}]$ ratio.

Our $\mathrm{Mg}_{2}$ indices are lower than those of $\mathrm{BC} 96$ and Vazdekis at high ages by less than $0.02 \mathrm{mag}$, but more close to those of Worthey. Probably due to the different calibrations for their indices, the $\mathrm{Mg}_{2}$ indices of Tantalo et al. are lower than those of all the other models considered here.

\section{Summary}

In this work we present Monte-Carlo evolutionary synthesis models for SSPs which cover a wide range in metallicity, from $Z=0.0001$ to $Z=0.05$, using most recent and complete sets of input physics: stellar evolutionary tracks for stellar masses from $0.08 M_{\odot}$ to $120 M_{\odot}$, including post helium flash evolution and mass loss, model atmosphere libraries also covering late stellar types and giving colours from $U$ to $K$ in agreement with observations and empirical calibrations for a series of absorption indices.

We obtain theoretical calibrations of colours and indices in terms of metallicity which for model ages of $10-15$ Gys agree closely with observations of GCs. The theoretical calibrations extend beyond the range of observed GCs, i.e. to a metallicity up to $[M / H] \leq 0.4$. Moreover, our models provide these theoretical calibrations for all ages from cluster formation to 15 Gyrs and thus can also be applied in the interpretation of young star cluster systems observed in many interacting and starburst galaxies.

The complete model files are available via WWW on http://www.uni-sw.gwdg.de/õkurth/ssp.html. There are also models with different parameters for the IMF and for mass loss available.

Acknowledgements. We thank Thibault Lejeune for providing us his library of theoretical stellar spectra, Isabelle Baraffe for providing us with her tracks for the low mass stars and our anonymous referee for valuable and instructive suggestions. This work was supported by the German Deutsche Forschungsgemeinschaft, DFG project number FR 916/3-1.

\section{References}

Ashman K.M., Zepf S.E., 1992, ApJ 384, 50

Bessell M.S., Brett J.M., 1988, PASP 100, 1134

Bessell M.S., Brett J.M., Scholz M., Wood P.R., 1989, A\&AS 77,1

Bessell M.S., Brett J.M., Scholz M., Wood P.R., 1991, A\&AS 89,335

Bressan A., Fagotto F., Bertelli G., Chiosi C., 1993, A\&AS 100,647 
Borges A.C., Idiart T.P., De Freitas Pacheco J.A., Thevenin F., 1995, AJ 110, 2408

Brodie J.P., Schroder L.L., Huchra J.P., Phillips A.C., KisslerPatig M., Forbes D.A., 1998, AJ 116, 691

Bruzual A.G., Charlot S., 1996 (in preparation) (BC96)

Burstein D., Faber S.M., Gaskell C.M., Krumm N., 1984, ApJ 287, 586

Buzzoni A., 1989, ApJS 71, 817

Cellone S.A., Forte J.C., 1996, ApJ 461, 176

Chabrier G., Baraffe I., 1997, A\&A 327, 1039

Chabrier G., Méra D., 1997, A\&A 328, 83

Contardo G., Steinmetz M., Fritze - v. Alvensleben U., 1998, ApJS 507

Covino S., Pasinetti Fracassini L.E., Malagnini M.L., Buzzoni A., 1994, A\&A 289, 775

Elson R.A.W., Santiago B.X., 1996, MNRAS 278, 617

Fagotto F., Bressan A., Bertelli G., Chiosi C., 1994, A\&AS 104,365

Fagotto F., Bressan A., Bertelli G., Chiosi C., 1994, A\&AS 105, 29

Fagotto F., Bressan A., Bertelli G., Chiosi C., 1994, A\&AS 105,39

Fluks M.A., 1994, A\&AS 105, 311

Forbes D.A., Brodie J.P., Grillmair C.J., 1997, AJ 13, 1652

Fritze - v. Alvensleben U., Gerhard O.E., 1994, A\&A 285, 751

Fritze - v. Alvensleben U., Burkert A., 1995, A\&A 300, 58

Fuhrmann K., Axer M., Gehren T., 1995, A\&A 301, 492

Gorgas J., Faber S.M., Burstein D., González J.J., Courteau S., Prosser C., 1993, ApJS 86, 153

Harris W.E., 1996, AJ 112, 1487

Kissler-Patig M., 1997, A\&A 319, 83
Kissler-Patig M., Brodie J.P., Schroder L.L., Forbes D.A., Gillmair C.J., Huchra J.P., 1998, A\&A 319, 83

Kurth O.M., 1998 (in preparation)

Kurucz R.L., 1979, ApJS 40, 1

Lejeune T., Cuisinier F., Buser R., 1998, A\&AS 130, 65

Lejeune T., Cuisinier F., Buser R., 1997, A\&AS 125, 229

Loxen J., Theis C., Fritze - v. Alvensleben U., Fricke K.J., Hensler G., 1992, Proceedings of the ESO/EIPC Workshop Structure, Dynamics And Chemical Evolution Of Elliptical Galaxies

Loxen J., 1997, Ph.D thesis Universität Göttingen

Miller B.W., Whitmore B.C., Schweizer F., Fall S.M., 1997, AJ 114,2381

Salaris M., Chieffi A., Straniero O., 1993, ApJ 414, 580

Schweizer F., Seitzer P., 1993, ApJ 417, 29

Schweizer F., Miller B.W., Whitmore B.C., Fall S.M., 1996, AJ 112,1839

Sweigart A.V., Gross P.G., 1978, ApJS 36, 405

Tantalo R., Chiosi C., Bressan A., Fagotto F., 1996, A\&A 311, 361

Tantalo R., Chiosi C., Bressan A., 1998, A\&A 333, 419

Whitmore B.C., Schweizer F., 1995, AJ 109, 960

Vazdekis A., Casuso E., Peletier R.F., Beckman J.E., 1996, ApJS 106, 307

Worthey G., Faber S.M., González J.J., Burstein D., 1994, ApJS 94, 687

Worthey G., 1994, ApJS 95, 107

Zepf S.E., Ashman K.M., 1992, MNRAS 264, 611

Zepf S.E., Carter D., Sharples R.M., Ashman K., 1995, ApJL 445,19 